\title{
TIME KINETICS OF BONE DEFECT HEALING IN RESPONSE TO BMP-2 AND GDF-5 CHARACTERISED BY IN VIVO BIOMECHANICS
}

\author{
Dag Wulsten ${ }^{1}$, Vaida Glatt ${ }^{1}$, Agnes Ellinghaus ${ }^{1}$, Katharina Schmidt-Bleek ${ }^{1}$, Ansgar Petersen ${ }^{1,2}$, Hanna Schell ${ }^{1,2}$, \\ Jasmin Lienau ${ }^{1,2}$, Walter Sebald ${ }^{3}$, Frank Plöger ${ }^{4}$, Petra Seemann ${ }^{2}$ and Georg N. Duda ${ }^{1,2}$ \\ ${ }^{1}$ Julius Wolff Institute and Center for Musculoskeletal Surgery, Charité - Universitätsmedizin Berlin, Germany \\ ${ }^{2}$ Berlin-Brandenburg Center for Regenerative Therapy, Charité - Universitätsmedizin Berlin, Germany \\ ${ }^{3}$ Theodor-Boveri Institute for Life Sciences (Biocenter), University of Wuerzburg, Germany \\ ${ }^{4}$ Biopharm GmbH, Heidelberg, Germany
}

\begin{abstract}
This study reports that treatment of osseous defects with different growth factors initiates distinct rates of repair. We developed a new method for monitoring the progression of repair, based upon measuring the in vivo mechanical properties of healing bone. Two different members of the bone morphogenetic protein (BMP) family were chosen to initiate defect healing: BMP-2 to induce osteogenesis, and growth-and-differentiation factor (GDF)-5 to induce chondrogenesis. To evaluate bone healing, BMPs were implanted into stabilised $5 \mathrm{~mm}$ bone defects in rat femurs and compared to controls. During the first two weeks, in vivo biomechanical measurements showed similar values regardless of the treatment used. However, 2 weeks after surgery, the rhBMP-2 group had a substantial increase in stiffness, which was supported by the imaging modalities. Although the rhGDF-5 group showed comparable mechanical properties at 6 weeks as the rhBMP-2 group, the temporal development of regenerating tissues appeared different with rhGDF-5, resulting in a smaller callus and delayed tissue mineralisation. Moreover, histology showed the presence of cartilage in the rhGDF-5 group whereas the rhBMP-2 group had no cartilaginous tissue.

Therefore, this study shows that rhBMP-2 and rhGDF5 treated defects, under the same conditions, use distinct rates of bone healing as shown by the tissue mechanical properties. Furthermore, results showed that in vivo biomechanical method is capable of detecting differences in healing rate by means of change in callus stiffness due to tissue mineralisation.
\end{abstract}

Keywords: Large segmental defects, bone healing, bone morphogenetic protein, growth and differentiation factor, small animal model.

\footnotetext{
*Address for correspondence:

Georg N. Duda

Julius Wolff Institute and Center for Musculoskeletal Surgery

Berlin-Brandenburg Center for Regenerative Therapies

Charité - Universitätsmedizin Berlin

Augustenburger Platz 1

D-13353 Berlin, Germany

Telephone Number: +49 30450559079

FAX Number: +4930450559969

E-mail: georg.duda@charite.de
}

\section{Introduction}

While the mechanical boundary conditions necessary for successful healing of fractures have been more-or-less unravelled, factors influencing healing of more complex fractures, such as critical size segmental defects (CSD) that fail to heal spontaneously, need further investigation. For decades the focus on defect healing has shifted towards identifying specific growth factors with potent osteogenic capabilities. Many different growth factors can produce successful bone repair in experimental animals. However, bone morphogenetic proteins (BMPs) are the only molecules known to be strong inducers of both bone and cartilage regeneration. Beginning with the earlier stages and continuing throughout bone healing, BMPs are produced by the mesenchymal stem cells (MSCs), osteoprogenitor cells, chondrocytes and osteoblasts. Indeed, it has been demonstrated that bone repair cannot even begin in the absence of BMP-2 in a mouse model (Tsuji et al., 2006). Initial studies showed that proteins extracted from the demineralised bone matrix (DBM) were able to induce bone formation at ectopic sites in rodents (Urist, 1965; Rosen, 2006). It was also described that the osteogenic process initiated by these extracted proteins closely resembled endochondral fracture healing and bone formation. Likewise, many years later, a study by Yasko et al. also reported that, in rats, CSD healed in response to BMP-2 treatment by endochondral ossification (Yasko et al., 1992).

Another member of the BMP family, BMP-14, which is known as growth and differentiation factor-5 (GDF-5), has also been evaluated in pre-clinical studies for cartilage repair and bone healing. It is thought to be acting mainly on MSCs, directing their differentiation into cartilage without directly converting the progenitor cells into osteoblasts during the process of endochondral bone formation in the skeletal development of limbs (Hotten et al., 1996). Conversely, there are conflicting reports suggesting that GDF-5 induces not only chondrogenesis, but also osteogenesis in vitro and in vivo (Wolfman et al., 1997; Erlacher et al., 1998a; Erlacher et al., 1998b; Gruber et al., 2000). Furthermore, that the carrier material in conjunction with other factors such as the dose has a significant influence on the type of tissue formed. All things considered, the factors underlying the process by which MSCs adopt one biologic pathway over the other in response to GDF-5 or BMP-2 is unclear, and what governs the predominant tissue outcome remains to be elucidated. 
Limited understanding of the interplay between mechanical and biologic factors is partly related to a lack of methods enabling the early detection of tissue formation at the organ level. Although new technologies improve not only the treatment of broken bones, but also our ability to monitor the progression of healing experimentally (Roberts and Steele, 2000; Hente et al., 2003; Thorey et al., 2008) and in clinical practice (Richardson et al., 1994; Seide et al., 2004), it is still not possible to differentiate in vivo the early effects on healing of either mechanical or biological stimuli. Even though there are many methods available for monitoring the progress of tissue regeneration at the molecular, cellular, structural and biomechanical levels, many investigations focus on the molecular and radiographic evaluation of bone healing. While these methods provide important information, most of them are in vitro evaluations that do not allow for temporal and serial monitoring of bone regeneration in vivo, furthermore, they require a large number of animals. In the last few years more and more studies have used in vivo quantitative $\mu \mathrm{CT}$ imaging to monitor the morphology of callus tissue. This technique is an excellent tool for the temporal monitoring of bone repair, but has its own limitations because it is only able to evaluate the mineralised phase of bone tissue, which is formed during the later phase of healing. Furthermore, this method is more expensive and more importantly exposes patients to higher amounts of radiation. Aside from the above mentioned side effects and disadvantages, the evaluations by these methods provide a more accurate measurement of bone density, which contributes to the mechanical properties of healing bone. However, monitoring by these devices does not provide information about the mechanical competence of healing callus tissue, which is a better determinant of bone tissue resistance to the applied mechanical forces through load bearing.

Only a small number of clinical and experimental studies have evaluated the healing of bone from the haematoma to the remodelling phase. These studies provide information about the natural bone healing, the influence of pharmaceuticals on bone healing and the influence of degradable implants on bone. In the majority of cases, histologic or histomorphometric evaluation was used to determine the properties of callus tissue in the early and later phases of bone healing. Despite being the methods of choice for both researchers and clinicians, these techniques cannot be performed in vivo. For many years researchers have attempted to develop alternative ways to determine the properties of healing bone in vivo. These methods include in vivo low-intensity pulsed ultrasound and biomechanical testing, which can measure the stiffness of the healing callus. Several in vivo stiffness methods have been described, but they often have limitations and weaknesses (Beaupre et al., 1983; Evans et al., 1988; Claes et al., 2002; Floerkemeier et al., 2005). The patients or animals were mostly awake during the procedure which might influence the measurements by uncontrolled movements of muscle contraction and this would have consequences on the accuracy of the stiffness calculations. In addition, some techniques used by others required complex finite element (FE)-evaluation or, difficult individual calculation methods, an individual calibration or complex measurement sensor technology. Thus it is difficult to use these methods to monitor bone healing or to detect the effect of different implants and drug therapies on bone regeneration, especially at the earlier phase.

For a full understanding of bone formation during the initial stage of bone repair, it is very important to be able to distinguish between distinct bone healing patterns in order to develop new therapies for specific tissue regeneration. Moreover, serial analysis of tissue formation and maturation initiated by different biological agents should help to identify differences in healing patterns resulting from each treatment. Such information could aid the discovery of new drug therapies for tissue regeneration, especially when the damage from an injury or disease is too severe for natural healing to occur. Therefore, we hypothesised that bone defect healing initiated by osteoinductive (BMP-2) and chondroinductive (GDF-5) growth factors would show distinct pathways of tissue regeneration and maturation that could be distinguished by the mechanical competence of callus tissue beginning at an early phase. Thus, the aim of the current study was to determine temporal changes and patterns of tissue formation and maturation in an in vivo bone defect rat model. To investigate the effect of different biological stimuli, we developed an in vivo biomechanical test that would allow for temporal monitoring of tissue regeneration as the bone defect healing progressed.

\section{Materials and Methods}

\section{Animal surgery and study design}

A total of twenty-four, 12 week old adult female Sprague Dawley rats (weights 250-300 g) were purchased from Charles River (Charles River WIGA Deutschland GmbH, Sulzfeld, Germany). For each animal, a $5 \mathrm{~mm}$ standardised mid-shaft critical sized segmental defect was created in the left femur (Mehta et al., 2011), and stabilised with a custom made external fixator. Briefly, an incision of approximately $3 \mathrm{~cm}$ was made through the skin running on the surface of the left femur from the supracondylar region of the knee to the greater trochanter. The femoral shaft of the left leg was exposed by gentle dissection. The template was placed on the anterior lateral aspect of the femur to guide the drill and permit reproducible positioning of four monocortical drill holes. During the drilling, saline irrigation was used to prevent heat damage to the bone tissue. Threaded, titanium bone pins (diameter $1.2 \mathrm{~mm}$ ) were driven into each hole until their tips reached the outer surface of the cortex. An external fixator was secured at the preset distance of $7.5 \mathrm{~mm}$ from the bone surface. Subsequently, guided by the template, a $5 \mathrm{~mm}$ defect was created using an oscillating saw. In order to minimise bone damage, $0.9 \%$ saline solution was applied during sawing.

Stable haemostatic collagen sponges (Lyostypt ${ }^{\circledR}, \mathrm{B}$. Braun, Melsungen, Germany) were used as a carrier to deliver growth factors. Under sterile conditions, sponges were cut into appropriately sized pieces $(10 \times 5 \times 5 \mathrm{~mm})$ 

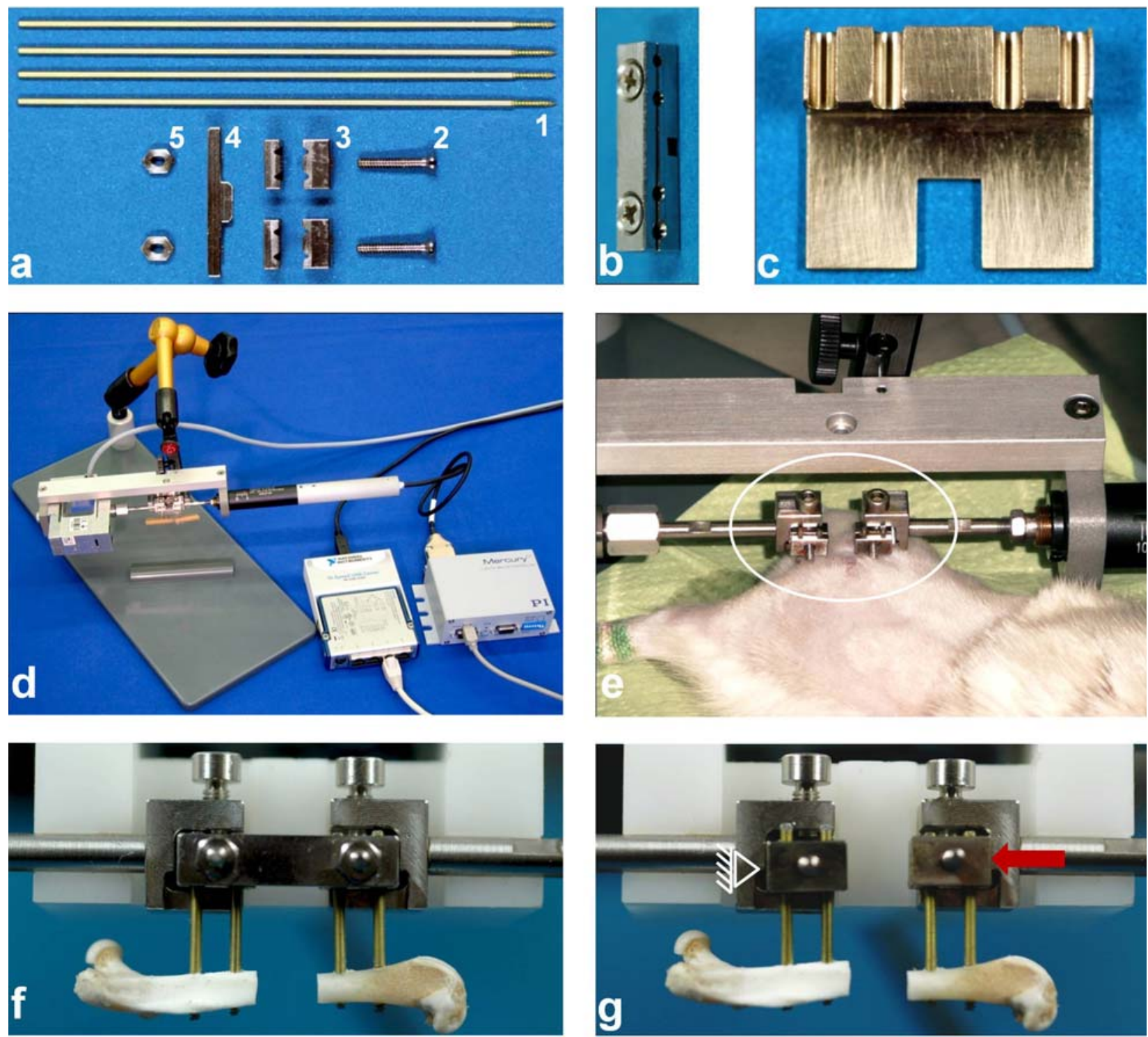

Fig. 1. (a) Components of custom made external fixator: 1-K-wires to fix the fixator bar to the bone; 2-screws used to secure the clamp and the cross bar to the clamps; 3-stainless steel pin clamps; 4-stainless steel cross bar; 4-nuts used to secure the cross bar to the clamps; (b) A template used to predrill four holes for reproducible placement of K-wires (c) Sawing template for reproducible osteotomy (d) The test setup, consisting of a linear actuator and a force sensor, are both fixed on to a U-frame. (e) The fixator mounted in the test setup for an in vivo measurement (fixator bar already removed) (f) The jig used to restrain the fixator for the in vivo biomechanical test with the cross bar still attached (g) The jig used to restrain the fixator for the in vivo biomechanical test with the cross bar removed before the measurement.

and rolled up to fit the defects used as vehicles to deliver BMPs. For the control group, Group $\mathrm{A}(\mathrm{n}=8)$, sponges were loaded with $50 \mathrm{ml} \mathrm{HCl}(10 \mathrm{mM} \mathrm{HCl})$ and implanted in the empty defects. One treatment group, Group B $(n=8)$, received $50 \mu \mathrm{g}$ of rhBMP-2 $\left(1 \mathrm{mg} / \mathrm{ml}, \mathrm{ddH}_{2} 0\right.$; Prof. Sebald, Würzburg, Germany) and the second treatment group, Group C ( $\mathrm{n}=8)$ received $50 \mu \mathrm{g}$ rhGDF5 $(2 \mathrm{mg} / \mathrm{ml}, 10 \mathrm{mM}$ $\mathrm{HCl}$ Biopharm, Heidelberg, Germany) to stimulate bone healing. Immediately after the sponge was inserted into the defect, the wound was closed in layers with muscle first and then the skin. After surgery, to have baseline data, defects in all the groups were subjected to an in vivo biomechanical test to determine the mechanical properties of empty gaps. Following this procedure, the rats were housed two per cage and were allowed to move freely. All animals were sacrificed six weeks after the surgery. All animal experiments were carried out according to the policies and procedures established by the Animal Welfare Act, the NIH Guide for Care and Use of Laboratory Animals, and the National Animal Welfare Guidelines. This study was approved by the local legal representative (LAGeSo, Berlin, Germany: G 0071/07).

\section{External fixator and mechanical characterisation}

A uniplanar unilateral custom made external fixation device was used to stabilise femoral critical size defect. The fixator consisted of four titanium Kirschner wires (Kwires, $1.2 \mathrm{~mm}$ diameter, $5 \mathrm{~mm}$ thread, Fig. 1a-1) and a fixator bar made out of stainless steel. The fixator bar was composed of a cross bar (Fig. 1a-4) and two pin clamps 
(Fig. 1a-3). Each clamp consisted of two tiny plates, where the thicker plate contained a threaded screw hole. The plates were bound by two countersunk screws (Fig. 1a-2,) in order to tighten either the proximal or the distal pair of $\mathrm{K}$-wires to the fixator. The titanium locking nuts were used to attach a cross bar to the clamps (Fig. 1a-5). The fixator was designed so that the cross bar could be repeatedly removed to allow an in vivo stiffness measurement in the defect gap. For the in vivo biomechanical test the fixator is restrained within the measurement setup to prevent dislocation in the osteotomy site (Fig. 1e, f, g). In order to restrain the fixator in the jig, the fixator clamps were secured with screws (Fig. 1f, g) and only then the cross bar was removed for an in vivo measurement. The jig was made out of stainless steel and has two identical parts included with two screws; one side was attached to a force sensor and the other side to a precision linear actuator (Fig. 1e). A drilling template was made to ensure reproducible placement of four K-wires (Fig 1b). The template is similar in material and dimensions to the actual fixator. Using the template, four $1 \mathrm{~mm}$ diameter bicortical holes were made by a drill bit followed by the insertion of K-wires through the template holes. A sawing template was also made to insure reproducible $5 \mathrm{~mm}$ osteotomy (Fig 1c).

A non-destructive in vitro biomechanical test was used to characterise the external fixators, which were specifically designed for this experiment. External fixators were mounted on six cadaveric femurs from 12-week-old female Sprague-Dawley rats to determine construct stiffness. Both ends of the femoral bones were embedded in custom-made casting containers with methylmethacrylate (Technovit 3400, Heraeus Kulzer, Hanau, Germany). The embedded specimens were mounted in a Bose universal testing machine (Bose ElectroForce LM1, Bose Corporation, Eden Prairie, MN, USA), with an applied preload of $0.3 \mathrm{~N}$, and tested in the axial compression mode. The specimens were compressed at a rate of $1 \mathrm{~mm} / \mathrm{min}$ until a maximum force of $10 \mathrm{~N}$ was reached. The measurement was repeated three times for each femoral construct, and the applied force and the recorded deformation parameters were used to calculate the stiffness of the fixation device.

\section{Radiographic evaluation (X-rays)}

Bone defect healing was evaluated by qualitative radiographic images using a mobile X-Ray device (Mobilett plus, Siemens, Munich, Germany). The X-Rays were taken at 2, 4, and 6 weeks post-surgery to determine the progress of defect healing in each group. While under general anaesthesia, each rat was placed in the ventral position and the left hind limb was rotated so the external fixator was not in the path of the X-ray source. All X-ray images were used only for qualitative purposes and were compared to the $3 \mathrm{D}$ in vivo $\mu \mathrm{CT}$ images from the same animal at the same time point.

\section{In vivo Micro-Computed Tomography}

Quantitative analysis of bone healing was assessed by an in vivo micro-computed tomography $(\mu \mathrm{CT})$ scanner (vivaCT 40, Scanco Medical, Bassersdorf, Switzerland) with a microfocus X-ray source at 2, 4, and 6 weeks postsurgery. Under general anaesthesia, all femurs were scanned using $35.5 \mu \mathrm{m}$ isotropic resolution, at $55 \mathrm{kVp}$ energy, $145 \mu \mathrm{A}$ intensity and $150 \mathrm{~ms}$ integration time. A volume of interest (VOI) was defined for the periosteal and endosteal callus, excluding the pre-existing cortical bone. The VOI included the $5 \mathrm{~mm}$ defect region and $0.5 \mathrm{~mm}$ in the proximal and distal directions from the borders of the original defect gap. A global threshold of $50 \%$ the mineral density of the intact tibia, equivalent to $351 \mathrm{mg} \mathrm{HA} / \mathrm{ccm}$ was used to distinguish mineralised tissue from poorly mineralised and unmineralised tissue. Outcome measures included mineralised callus volume (bone volume, $\mathrm{BV}, \mathrm{mm}^{3}$ ), total callus volume $\left(\mathrm{TV}, \mathrm{mm}^{3}\right.$ ), callus mineralised volume fraction (BV/TV), bone mineral density (BMD, mg HA/ccm), tissue mineral density (TMD, mg HA/ccm) and bone mineral content (BMC, mg HA), defined as BV multiplied by BMD. BMD was calculated using only the voxels whose intensity exceeded the global threshold.

\section{Biomechanical in vivo test}

A new biomechanical testing method was developed to determine the stiffness of callus tissue in vivo during healing. Stiffness was measured immediately after the surgery, and then monitored at weekly intervals. In vivo measurements were carried out under general anaesthesia, all animals were injected intraperitoneally with $0.3 \mathrm{mg} / \mathrm{kg}$ medetomidine (Domitor ${ }^{\circledR}$, Pfizer, Karlsruhe, Germany) and $60 \mathrm{mg} / \mathrm{kg}$ ketamine (Ketamin-ratiopharm ${ }^{\circledR}$, Ratiopharm, Ulm, Germany). The newly developed adjustable test setup consisted of a metallic U-frame with an attached force sensor (S2/20N, Hottinger Baldwin Messtechnik, Darmstadt, Germany) on one side, and a linear precision actuator (M-230, Physik Instrumente, Karlsruhe, Germany) on the opposite side, controlled by a self developed Labview script (LabVIEW 8.5, National Instruments, Austin, TX, USA). Moreover, the external fixator (described in the previous section) used to stabilise a $5 \mathrm{~mm}$, CSD was custom designed in such a way that the stiffness properties of healing callus could be measured by removing the stability bar. For every measurement the proximal side of the external fixator was attached to the load cell and the distal side was attached to the linear precision actuator. This prevented dislocation of the CSD cortical edges (Fig. 1a). The stability bar of the fixator was then detached (Fig. 1b), thereby permitting only a mono axial compression deformation. To measure the force in the defect gap a set protocol was used, which consisted of a compression phase with a $500 \mu \mathrm{m}$ displacement applied at a constant rate of $10 \mu \mathrm{m} / \mathrm{s}$, followed by a resting phase of $40 \mathrm{~s}$, where the compression was kept constant (Fig. 2). Finally, the linear actuator returned to the initial position at the same rate and rested for $40 \mathrm{~s}$. The whole procedure was repeated five additional times, while continuously recording the axial reaction force. Due to the biological nature of callus tissue, the initial 3 cycles were used for preconditioning and only the latter 3 were included in the data analysis.

The parameters analysed for each specimen were obtained from the elastic compression phase and from the 


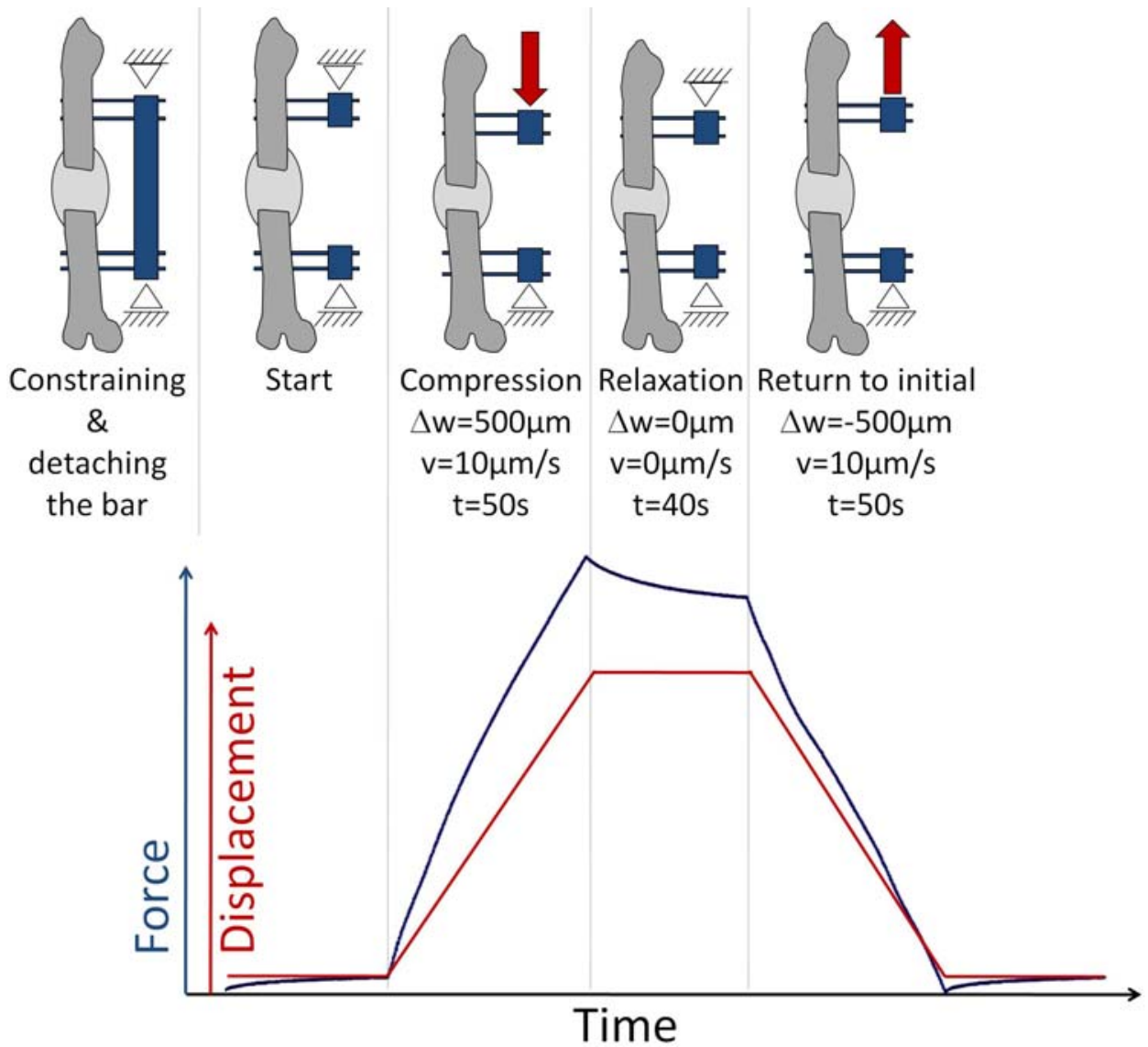

Fig. 2. The external fixator on the proximal side was attached to the load cell and the distal side to the linear precision actuator. The stability bar of the fixator was then detached, thereby permitting only a mono axial compression deformation by measuring 6 ramp force relaxation tests at each time point. Then the linear actuator was returned to its initial position and rested for $40 \mathrm{~s}$. The entire procedure was repeated 5 additional times. Only the last 3 cycles were used for data analysis.

relaxation phase using the force and displacement data. The maximal reaction force was calculated as the difference between the force value at the beginning of the compression phase and at the peak force at the end of the compression phase. The stiffness of the callus tissue, defined as a measurement of an elastic body to resist deformation by an applied force, was calculated from the linear portion of load-displacement curve. The relaxation force, derived from the second part of the measurement cycle, at the time when the applied displacement was kept constant for $40 \mathrm{~s}$, was used in order to determine the viscoelastic characteristics of callus tissue. It should be noted that not all of the measurements reached full relaxation equilibrium within this time frame. The relaxation force was calculated as the difference between the averaged first ten points of the force at the end of the compression phase, and the averaged last ten points of the resting phase, which is where the force started to plateau. The relative relaxation force was calculated as the ratio between maximal reaction force applied during compression and the relaxation force while in the resting phase. The relaxation force refers to the reduction in compression as a result of movement or rearrangement within the non-mineralised soft tissue network that gives the callus its visco-elastic behaviour. However, the relaxation force measurement is also heavily dependent upon the prior applied force. Over the course of healing, maturation of the callus tissue induce changes in force, and since a preset displacement was used to apply the force the applied force varied depending on the test group and the time point measured as the type of callus tissue went from viscous to visco-elastic and then to a more elastic material. In order to compare the actual properties (viscosity) of the callus tissue between the groups as the temporal changes occurred, the relaxation force measurements in each group and for the each time point needed to be normalised. The resulting parameter is called the relative relaxation force. The relevance of this parameter is that it eliminates the influence of the varied forces measured, which was different depending on the healing phase, and only reflects the elastic nature of callus tissue as the defect healing progressed. Hence, the higher is the relative relaxation force the more flexible callus tissue is measured in the defect gap, which suggests cartilaginous not mineralised tissue material.

\section{Histology}

After sacrifice of animals at 6 weeks, specimens were decalcified in EDTA. Fixed and decalcified tissues were dehydrated in graded ethanol up to $100 \%$, transferred to xylol, and embedded in paraffin. Four-micron paraffin sections were cut in the frontal plane and placed on poly L-lysine coated slides, dried overnight and evaluated 
a

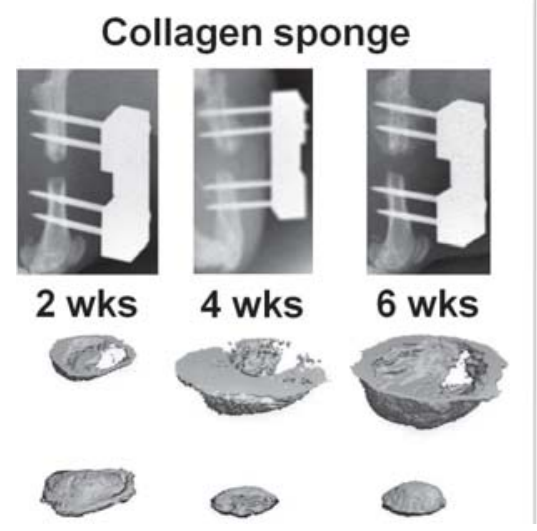

b

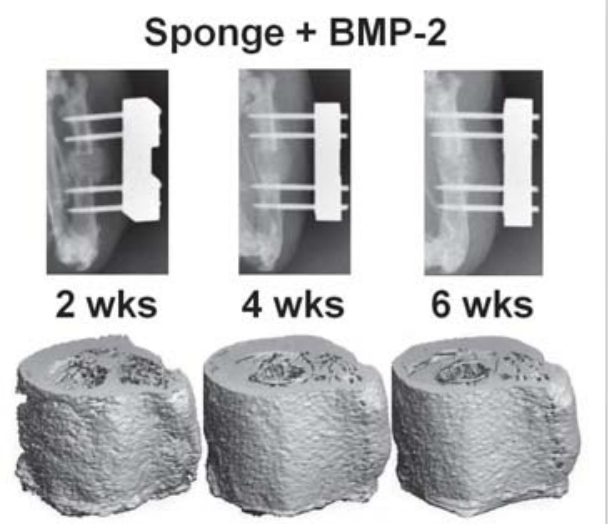

C

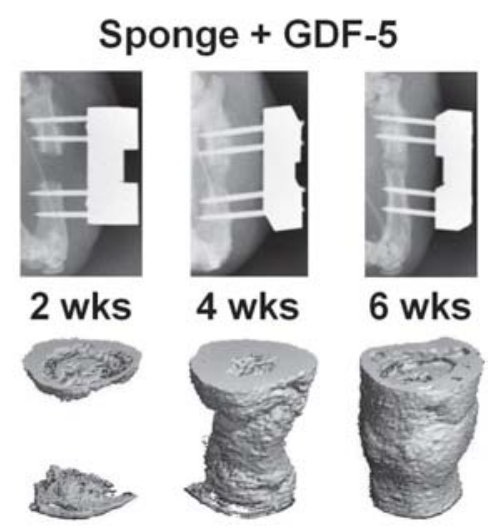

Fig. 3. Representative radiographic and 3D in vivo $\mu \mathrm{CT}$ images demonstrating repair of femoral segmental defects induced by rhBMP-2 and rhGDF- 5 and compared to the control group at 2, 4 and 6 weeks.

immediately or stored at $4^{\circ} \mathrm{C}$. Sections were stained with Movat's Pentachrome stain to determine the overall quality of callus tissue.

\section{Statistical analysis}

All collected data were averaged for each in vivo $\mu \mathrm{CT}$ and biomechanical test measurement. They were reported as the median value, and the upper and the lower quartile for each treatment group and time point, which is represented by a vertical line in the graphs. The assumption of normality was tested using the Shapiro-Wilk normality test. To determine statistical differences at each specific time point during bone healing between the three groups, a parametric pair wise $t$-test was performed, followed by a posthoc Bonferroni $p$-value adjustment multiple comparison procedure. Repeated measures ANOVA was used to analyse the overall temporal changes and the effect of treatment on bone repair. In addition, temporal changes between any two time points within one group were also analysed using a simple $t$-test. Differences were considered significant for a $p$-value of less than 0.05 . All test were performed using the SAS statistical software (SAS 9.1, Cary, NC, USA).

\section{Results}

\section{Characterisation of fixation device}

The axial stiffness evaluation was performed on the customized fixator constructs, mounted on the six cadaveric femurs. From every measurement, the applied forces and recorded deformations were used to calculate the average fixator stiffness for each construct combination. The results showed that the axial stiffness measurement of the femoral constructs had a mean value of $62( \pm 15) \mathrm{N} / \mathrm{mm}$.

\section{Radiographic evaluation (X-Rays)}

Biweekly X-rays (Fig. 3) revealed that bone callus size was biggest in the group treated with rhBMP-2 (b) at all time points. Furthermore, early mineralisation and complete bridging of callus was seen 2 weeks after surgery, but only in the group with rhBMP-2 treatment. The control group (a) and the rhGDF-5 group (c), in contrast, had no evidence of bone formation in the defect gap at the same time point. However, by week four all treated defects were bridged regardless of the growth factor, with the biggest callus being in the group receiving rhBMP-2. By the end of the experiment, which was 6 weeks after the surgery, the callus size in the rhGDF-5 group appeared larger than it was at 4 weeks. In contrast, X-rays showed that the group treated with rhBMP-2 had no change in callus size at the end of the study, but was clearly larger than in the rhGDF-5 group. No bone formation was observed by X-ray in the control group, at any time point.

\section{In vivo Micro-Computed Tomography}

Quantitative analysis of the in vivo $\mu \mathrm{CT}$ data confirmed the radiographic images (Figs. 3 and 4). Two weeks after surgery, bone volume (BV) in the rhBMP-2 group was significantly higher than the control and rhGDF-5 groups (rhBMP-2 vs. Control, $p<0.001$; rhBMP-2 vs. rhGDF5, $p<0.001$; Fig. $4 a)$. It is worth noting that at this time point in the rhGDF-5 group the only evidence of bone formation, with the appearance of periosteal callus, was seen adjacent to the edges of the defect on the proximal and distal sides. In contrast, there was no bone formation in the control group. By week four the group treated with rhGDF-5 demonstrated a significant increase in $\mathrm{BV}$, and the amount of new bone continuously increased until 6 weeks postsurgery as indicated by bigger callus size (change 2-4 weeks, $p=0.001$ ). However, at no time point did the rhGDF-5 group have more BV than the rhBMP-2 group, and the values were significantly different for each time point (rhBMP-2 vs. rhGDF-5, 2 weeks $p<0.001$, 4 weeks $p<0.001,6$ weeks $p<0.005$ ). Interestingly, in the rhBMP2 group, a very high amount of BV in the defect was reached at 2 weeks post-surgery, which then slightly increased until week 4 and then remained constant towards the end of the study (change 2-6 weeks, $p=0.489$ ). The control group had no bone formation in the defects at any time point, except adjacent to the proximal edge of the defect where small periosteal callus was seen. A statistically significant difference in BV was not observed only between the control and the rhGDF- 5 group at the 2 weeks time point (control vs. rhGDF-5, $p>0.999$ ). 

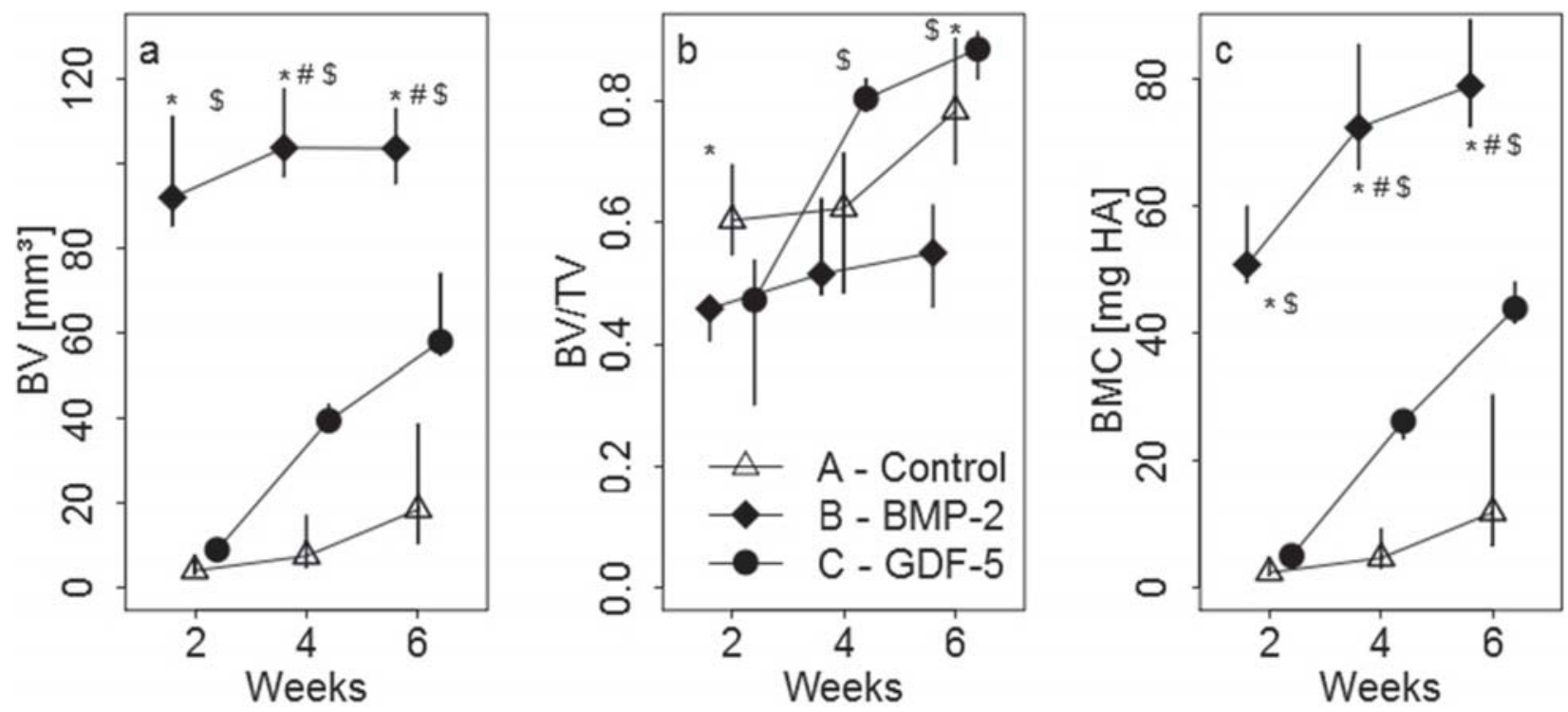

Fig. 4. In vivo $\mu \mathrm{CT}$ was used to measure bone volume $\left(\mathrm{BV}, \mathrm{mm}^{3}\right.$, (a), bone volume fraction $\left(\mathrm{BV} / \mathrm{TV}, \mathrm{mm}^{3},(\mathbf{b})\right.$ and bone mineral content (BMC, mg, (c). The results are reported as median values with upper and lower quartile for each group and time point. Asterisks indicate statistically statistical difference between the control and rhBMP-2 groups. Hash signs indicate significant differences between the control and rhGDF-5 groups. Dollar signs indicate significant differences between the rhBMP-2 and rhGDF-5 groups $(p<0.05)$.

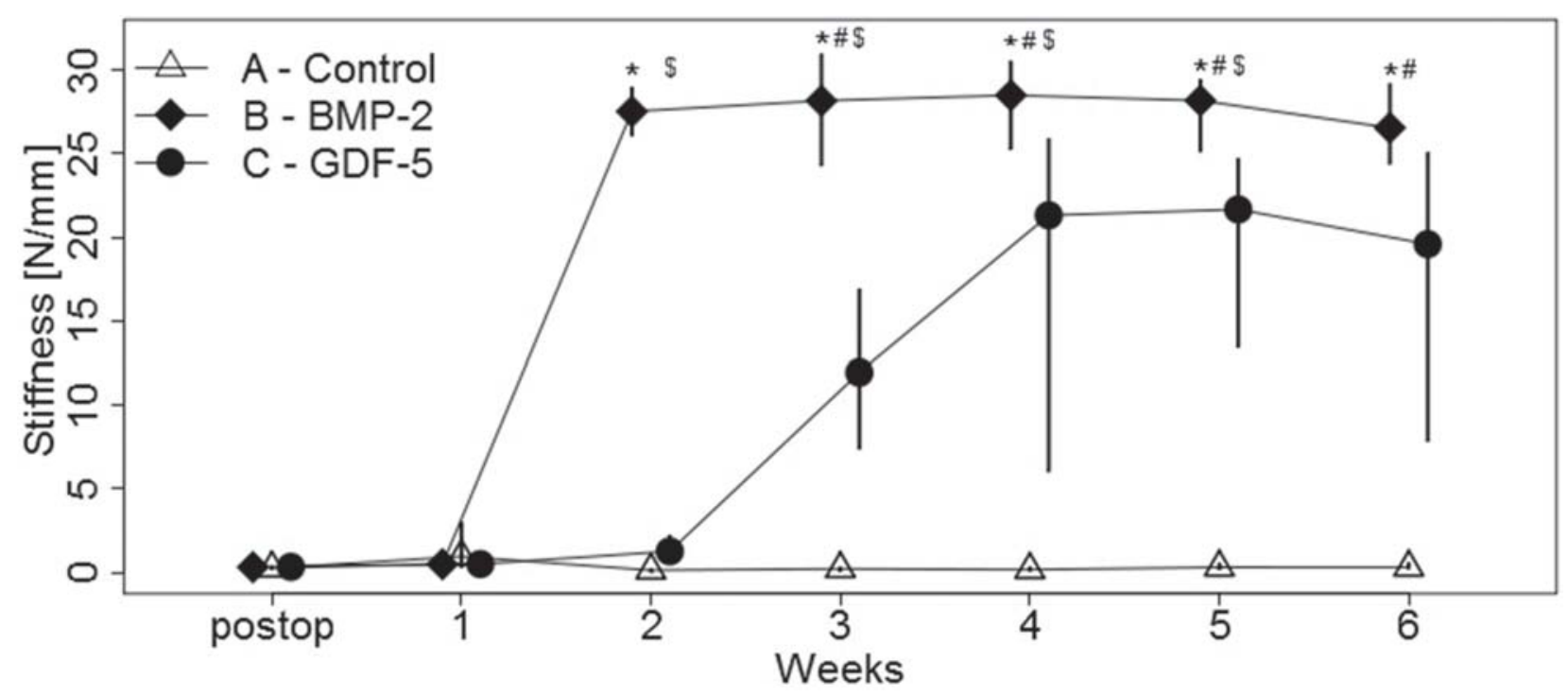

Fig. 5. Weekly in vivo biomechanical tests were used to determine callus stiffness in the defect gap over the course of bone healing in the control group (a); the rhBMP-2 treated group (b); and the rhGDF5 treated group (c). Results are reported as medians with upper and lower quartiles for each group and time point. Asterisks indicate statistically significant differences between the control and rhBMP-2 groups. Hash signs indicate significant differences between the control and rhGDF-5 groups, and dollar signs indicate significant differences between the rhBMP-2 and rhGDF5 groups $(p<0.05)$.

Bone volume fraction (BV/TV) in the rhBMP-2 group increased slightly between 2-6 weeks but this was not statistically significant ( $p=0.057$ Fig. $4 b$ ). However, in the rhGDF-5 group the increase in BV/TV between 2 and 4 weeks was statistically significant $(p=0.008)$ and continued to increase until 6 weeks. In the control group between 2 and 6 weeks a change in BV/TV values was also noted and found to be significantly different ( $p=0.041$, Fig. $4 b$ ). It is important to note that at no point during the study did the defects in the control group have any bone formation, and the only bone formed was adjacent to the defect to cap the edges of the broken bones.

Bone mineral content (BMC) had similar trend in all the groups with BV (Fig. 4a and c). The group treated with rhGDF-5 exhibited a steady increase in BMC, which increased to more than 3 times the value from 2 to 6 weeks $(p<0.001)$, and by week 6 reached values similar to those noted in the rhBMP-2 group (Fig. 4c). In the control group, BMC slightly increased from 2 to 6 weeks, but this increase was statistically significant $(p=0.049)$. The rhBMP-2 group 


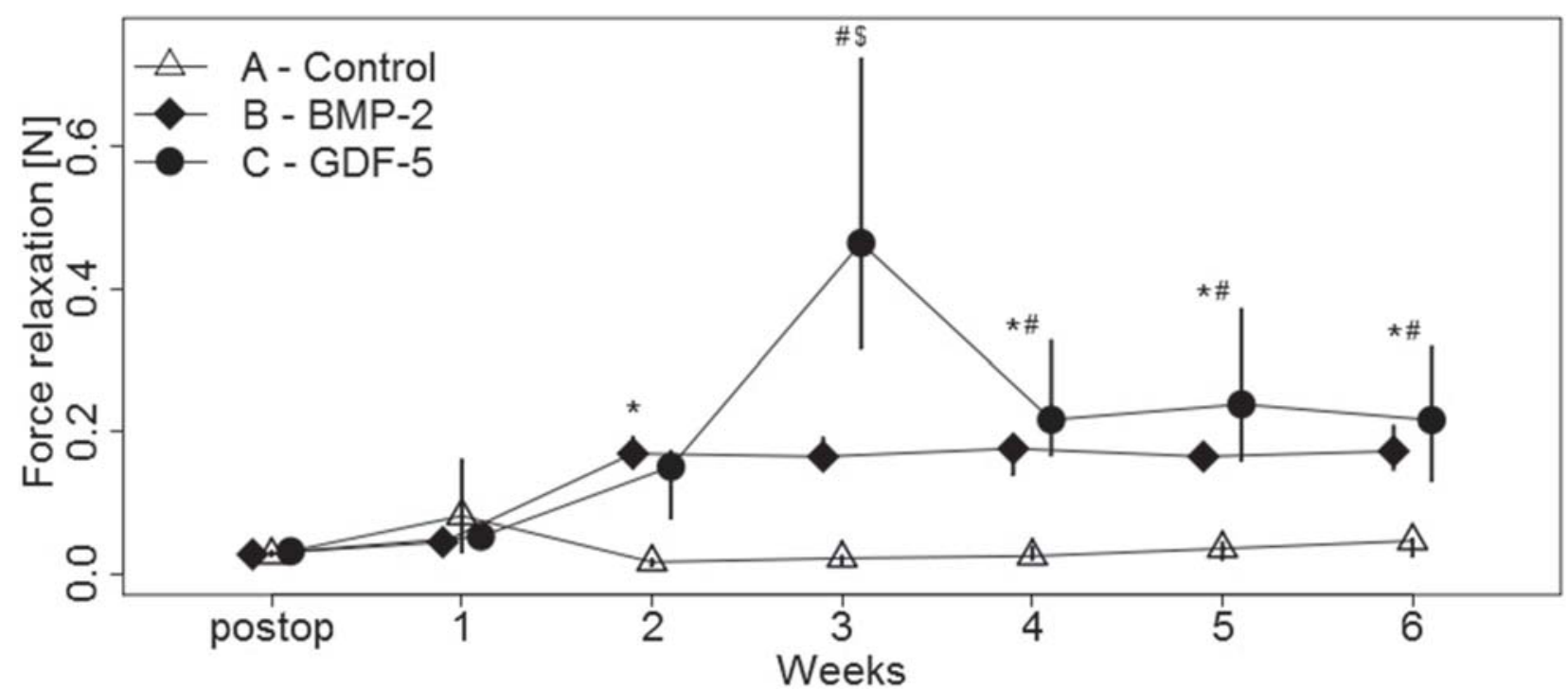

Fig. 6. Weekly in vivo biomechanical tests were used to determine the relaxation force in the defect gap over the course of bone healing in the control group (a); the rhBMP-2 treated group (b); and the rhGDF5 treated group (c). Results are reported as medians with upper and lower quartiles for each group and time point. Differences in relaxation force represent the viscoelastic characteristics of callus tissue. Asterisks indicate statistically significant differences between the control and rhBMP-2 groups. Hash signs indicate significant differences between the control and rhGDF-5 groups, and dollar signs indicate significant differences between the rhBMP-2 and rhGDF-5 groups $(p<0.05)$.

showed large increase in BMC from 2 to 4 weeks, while only a slight change was seen from 4 to 6 weeks. At 2 weeks after the surgery there was no difference in BMC between the rhGDF-5 and the control group (control vs. rhGDF-5, $p>0.999$ ). In contrast, a significant difference was seen when both groups where compared to the rhBMP2 group (rhBMP-2 vs. rhGDF-5, $p<0.001$, rhBMP-2 vs. control, $p<0.001)$. However, at 6 weeks BMC showed statistically significant difference within all the groups (rhBMP-2 vs. rhGDF-5, $p<0.002$, rhBMP-2 vs. control, $p<0.001$, control vs. rhGDF-5, $p=0.013$, Fig. 4c).

It is important to note, that the overall temporal changes analysed by repeated measures ANOVA was significantly different for all parameters and groups, except for the BV/ TV measurement between the control and rhGDF-5 groups.

\section{Biomechanical in vivo analysis}

Fig. 5 shows that the stiffnesses measured in defects by the in vivo biomechanical test one week after surgery were low and not different between the groups. However a week later, which was 2 weeks after the initial surgery, stiffnesses in the defects treated with rhBMP-2 had significantly increased and remained relatively unchanged as the bone healing progressed to 6 weeks after the surgery (change 2-6 weeks $p=0.7$ ). On the contrary, defects treated with rhGDF-5 treatment had no change after 2 weeks post surgery. However, a week later the stiffnesses in the defects substantially increased and continued to increase until 4 weeks when it plateaued, remaining unchanged until the end of the study (change 4-6 weeks, $p=0.073$ ). In both treatment groups, the stiffness peak values were reached within 4 to 6 weeks. Although only at 6 weeks no significant differences were observed between the treated groups, although the stiffnesses in the rhBMP-2 group tended to be higher (rhBMP-2 vs. rhGDF-5, 6 weeks $p=0.056$ ). In the control group, defect stiffness had practically no change throughout the study period (change 2-6 weeks, $p=0.531$ ) and was close to the lowest detectable values of the force sensor in the mechanical testing system. While there was no change in the stiffness values a week after the surgery between each group, a week later (which was 2 weeks after surgery) the rhBMP-2 group had a greater than 100-fold increase as compared to the other groups (rhBMP-2 vs. rhGDF-5, $p<0.001$, rhBMP-2 vs. control, $p<0.001)$. Yet, three weeks after surgery the stiffness values remained the same in the BMP-2 and control groups. However, the rhGDF-5 group showed a clear increase in stiffness at 3 weeks after surgery, and this was significant when compared to all other groups (rhGDF-5 vs. rhBMP-2, $p<0.001$; rhGDF-5 vs. control, $p<0.001$, Fig. 5). There was a steady increase in stiffness values between 2 and 4 weeks followed by a slight change from 4 to 6 weeks.

One week after surgery, the relaxation forces (RF) in all the groups had slightly increased, but differences between groups were not statistically significant (rhBMP2 vs. rhGDF-5, $p>0.999$, rhBMP-2 vs. control, $p=0.221$ control vs. rhGDF-5, $p=0.167$, Fig. 6). In the rhBMP-2 group, the RF significantly increased between weeks 1 and 2, and remained unchanged until the end of the study (change 1-2 weeks, $p=0.003$ ). The rhGDF-5 group followed the same trend as rhBMP-2 group until week 2 . In contrast, by week 3 the RF had a significant, 3fold increase in the rhGDF-5 group (change 2-3 weeks, $p=0.019$ ). Interestingly, by week 4 the relaxation force values in the rhGDF-5 group declined and were similar to those in the rhBMP-2 group. The control group had a slight 


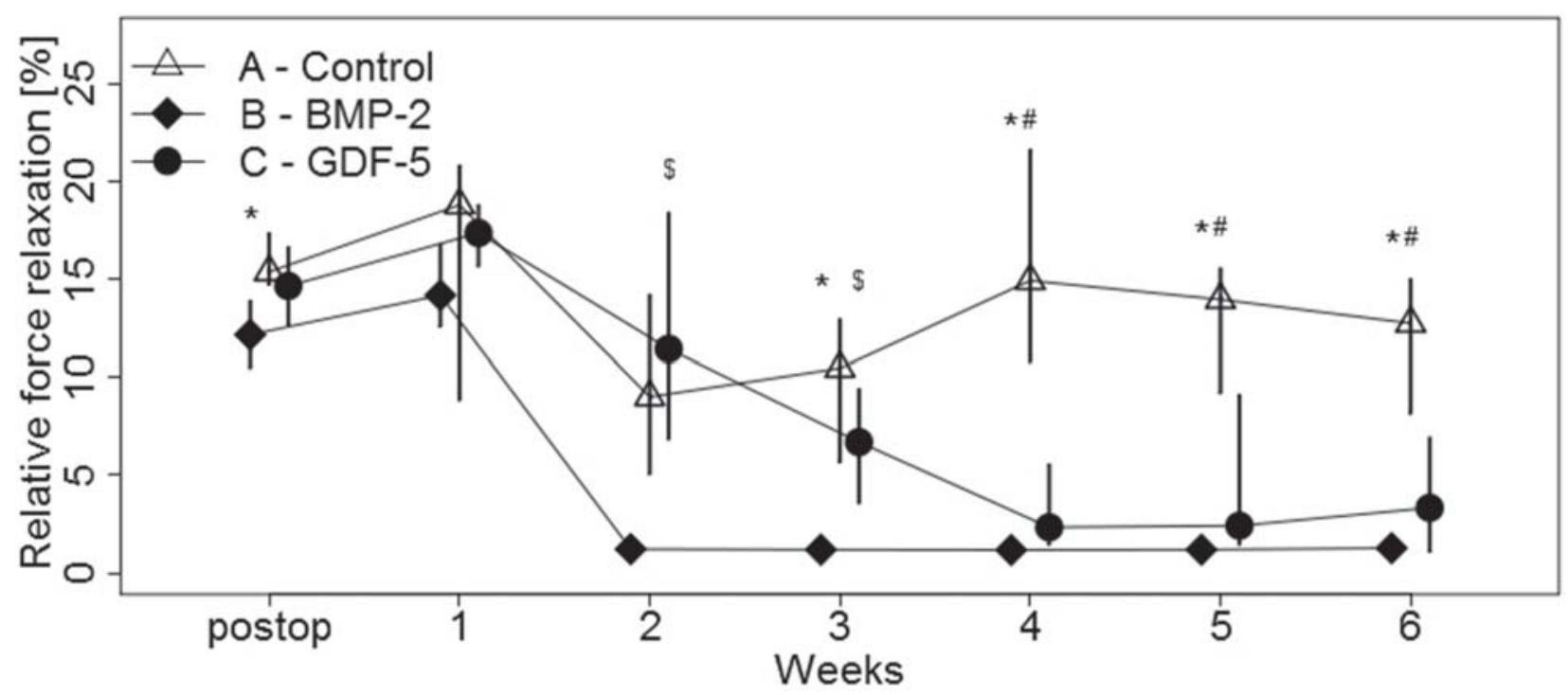

Fig. 7. Weekly in vivo biomechanical tests were used to determine the relative relaxation force in the defect gap over the course of bone healing in the control group (a); the rhBMP-2 treated group (b); and the rhGDF5 treated group (c). The relative relaxation force was calculated as the ratio between maximal reaction force applied during compression and the relaxation force while at rest. The results are reported as medians with upper and lower quartiles for each group and time point. Asterisks indicate statistically significant differences between the control and rhBMP2 groups. Hash signs indicate significant differences between the control and rhGDF-5 groups, and dollar signs indicate significant differences between the rhBMP-2 and rhGDF-5 groups $(p<0.05)$.

decline in RF between 1 and 2 weeks, and then remained steady until the end of the study. However, a week later the RF in the rhBMP-2 group was significantly different from the control group, but had no difference compared to the rhGDF-5 group (rhBMP-2 vs. rhGDF-5, $p=0.271$, rhBMP-2 vs. control, $p=0.001)$. In contrast, there was a significant difference between each group at 3 weeks after surgery with the highest values in the rhGDF-5 group, while there was no significant difference between the rhBMP-2 and the control group (rhBMP-2 vs. rhGDF-5, $p=0.003$, rhBMP-2 vs. control, $p=0.137$, control vs. rhGDF-5, $p<0.001$, Fig. 6).

One week after surgery, the relative relaxation force (RRF) followed the same trend and was similar in all groups (Fig. 7). At the second week, the RRF showed a similar pattern in every group by having a large decrease from week one. While significant change was only observed in the control and rhBMP-2 groups, this decrease was more pronounced in the rhBMP-2 group (change 1-2 weeks, rhBMP-2, $p<0.001$, control, $p=0.011$, rhGDF-5, $p=0.1$ ). In addition, the RRF values in the rhBMP-2 group were very low and did not change from 2 to 6 weeks. The group treated with rhGDF-5 had a gradual decrease in RRF values from 1 to 4 weeks, when it levelled off to the values of the rhBMP-2 group and remained constant until the end of 6 weeks. Conversely, from 3-4 weeks a slight increase in the RRF was observed in the control group, but remained steady from 4-6 weeks.

In vivo biomechanical data revealed similar results to $\mu \mathrm{CT}$ data when was analysed by the repeated measures ANOVA. The results showed that statistically significant change was seen for the overall temporal change as the defect healing progressed in all the groups.

\section{Histology}

Qualitative findings from the histological analysis were consistent with the imaging data (Fig. 8). A Movat Pentachrome stain was used to identify the presence of different tissues in the healed defect: fibrous tissue stained in black-grey and light pink, muscle tissue stained red, chondral tissue stained in green and the bone appeared yellow. It revealed differences between the groups in the composition of callus tissue at the end of 6 weeks of treatment.

In the control group (a), the defects had no evidence of bone formation. Instead, a mineralised encapsulation was seen on the pre-existing cortical edges of the proximal as well as the distal sides of the defect, while the intermediate gap had loosely organised fibrous tissue (Fig. $8 \mathrm{a}$ and $\mathrm{b})$. In contrast, there was considerable amount of bone formation in the defects treated with rhBMP-2 and rhGDF-5 (Fig. 8c and e, respectively), although the morphology of callus differed between the groups. For instance, in the rhBMP-2 group (Fig. 8c), a thin new periosteal cortical callus bridged the defect spanning from distal to the proximal side, while a double cortical callus was seen on the opposite side, this phenomenon was seen for every specimen. Although in the GDF-5 group less pronounced double cortical callus was only seen on one proximal side of the defect edge extending towards the femoral head. Furthermore, the defect gap contained a moderate amount of trabecular bone. In contrast, a histological section from the rhGDF-5 group also formed a considerable amount of bone, but this was less well organised. The neocortices were not clearly distinguishable and had no real callus. Although a large amount of new bone was detected within the defect, formed bone appeared more solid with lower porosity suggesting delayed 

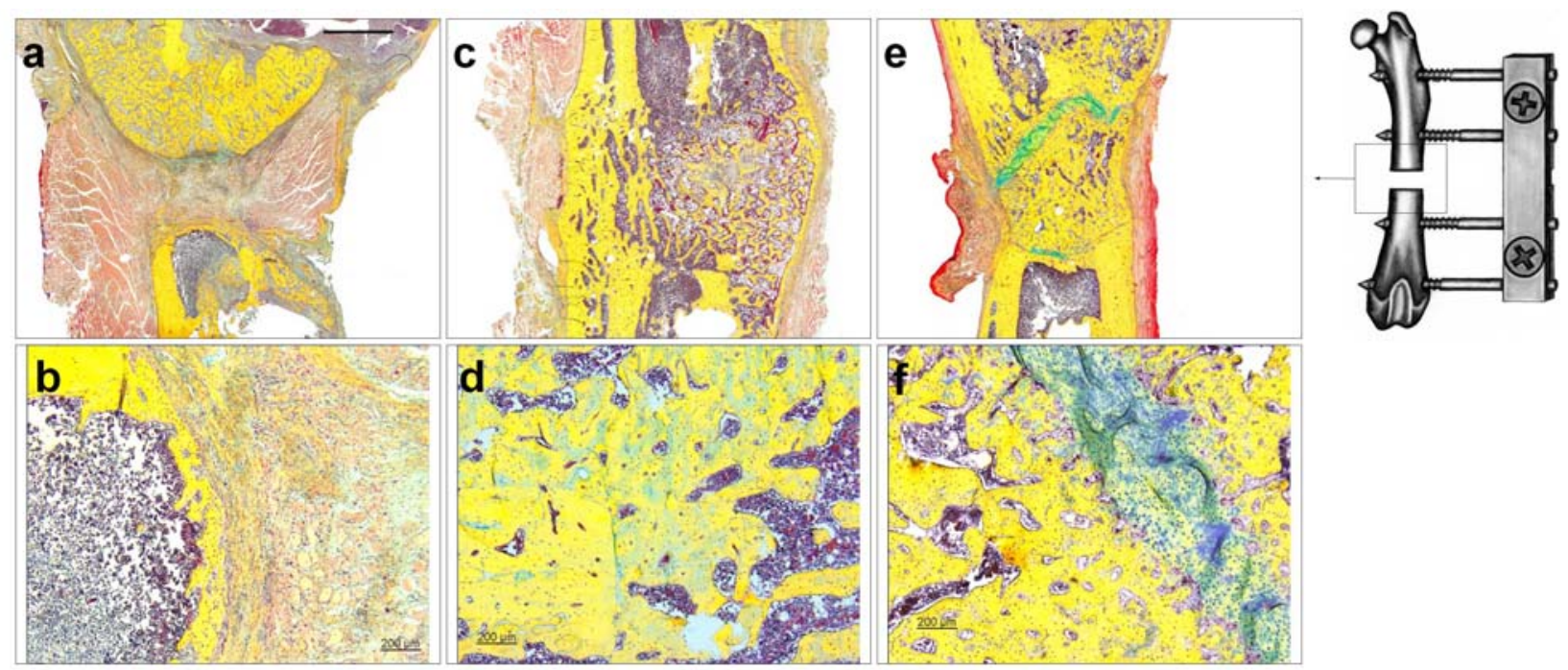

Fig. 8. Representative histological longitudinal sections from the femoral segmental defects 6 weeks post surgery in 3 different groups: (a, b) Control; (c, d) rhBMP-2 treatment; (e, f) rhGDF-5 treatment. Paraffin embedded sections stained with Movat Pentachrome show the general appearance of tissues within the defect: fibrous tissue stains black-grey and light red, muscle stains red, cartilage stains green and mineralized tissue stains yellow. Scale bar is $2 \mathrm{~mm}$ or $200 \mu \mathrm{m}$.

remodelling (Fig. 8e). Furthermore, the rhGDF-5 group had persistent cartilage as shown by the green staining (Fig. 8e and f), which could not be seen in the X-ray and $\mu \mathrm{CT}$ images. This suggested that the process of healing was initiated through endochondral ossification. The group with the rhBMP-2 treatment showed no cartilage formation (Fig. 8c). However, at the high magnification, histological images showed small traces of pale blue-green staining on the cortical bone adjacent to the defect and along the trabecular bone surface in the defect gap, which indicates remodelling phase in this group (Fig. 8d).

\section{Discussion}

Among all the different growth factors that are known to be involved in bone regeneration, bone morphogenetic proteins (BMPs) are the only molecules that are clinically used to enhance bone healing. Despite the knowledge that BMPs are strong inducers of osteogenic and chondrogenic tissues, the functions of different BMPs during in vivo tissue regeneration, and factors that determine biologic pathways of tissue formation and maturation patterns are not fully elucidated and require further examination. Therefore, the main goal for this study was to compare critical sized defect healing in rats treated with rhBMP-2 and rhGDF-5 in vivo, and to determine if bone tissue formation and maturation follow distinct biologic pathways. Furthermore, to help identify distinct healing pathways occurring in the earlier and later stages of healing in the different treatment groups, we developed an in vivo biomechanical test that permits temporal monitoring of tissue regeneration as the bone healing progresses.

The current study confirms that the pattern of bone healing in critical sized defects is different between rhBMP-2 and rhGDF-5 treatments when provided with the same local mechanical environment, dose and the same carrier. In the rhBMP-2 group early ossification occurred, whereas in the rhGDF-5 group a prolonged chondrogenic phase was observed. In fact, the stiffnesses of the defect tissues, as measured by our in vivo biomechanical test, revealed that 2 weeks after surgery the group that had the rhBMP-2 treatment had a significant increase in callus stiffness when compared to the rhGDF-5 group. Furthermore, one week later there was no change in callus stiffnesses in the defects treated with rhBMP-2. In contrast, defects treated with rhGDF-5 had a significant increase in callus stiffness at 3 weeks, which continued to increase until week four and remained the same until the end of the study. Likewise, the relative relaxation force, a measure reflecting the elastic nature of callus tissue as the defect healing progressed, was also significantly higher from 2 to 4 weeks in the rhGDF-5 group which suggests the presence of cartilaginous and non mineralised material in the defect gap. Furthermore, the histological analysis at the end of study showed the presence of residual cartilage in the rhGDF-5 group, while the rhBMP-2 group had no cartilaginous tissue. All together these findings clearly indicate that the defects treated with rhBMP-2 and rhGDF5 had different rates of tissue formation and maturation as bone regeneration progressed. On the other hand, these data may also imply that exogenous rhGDF-5 treatment heals defects via classical endochondral ossification, while the defects enhanced with rhBMP-2 may heal through intramembranous ossification. In fact, these observations are supported by a number of reports in the literature where it is demonstrated that bone repairs with exogenous rhGDF-5 treatment initiated endochondral ossification (Erlacher et al. 1998b; Bai et al. 2004; Kakudo et al. 2007; Kadomatsu et al. 2008). At the same time, other studies from the literature have reported that chondrogenic and osteogenic lineage pathways in response to rhGDF-5 treatment are dose and carrier dependent (Spiro et al. 2000; Shimaoka et al. 2004; Kakudo et al. 2007; Kadomatsu et 
al. 2008). However, to confirm this theory histological analysis needs to be performed at the earlier time points to determine if the same or different biological pathways are used.

Data obtained from in vivo biomechanical testing were also supported by additional imaging analyses. The radiographic $2 \mathrm{D}$ X-rays and in vivo $\mu \mathrm{CT} 3 \mathrm{D}$ images obtained in this study also demonstrated that the type of BMP used could have a differential effect on tissue formation and maturation, especially in the initial stages of bone healing. Both imaging modalities confirmed that there was no bone formation in defects with rhGDF-5 treatment two weeks after surgery. In contrast, defects treated with rhBMP-2 presented with a big callus and a large amount of new bone after two weeks of treatment. Interestingly, four weeks after surgery the defects treated with rhGDF-5 were completely filled with new bone, although the callus size was significantly smaller than that of the rhBMP-2 group. As the healing progressed until 6 weeks after surgery, the callus size and the amount of new bone increased in rhGDF-5 group, however in the rhBMP2 group it remained the same. Furthermore, the bone mineral content as assessed by $\mu \mathrm{CT}$, showed that the rhBMP-2 group had more advanced mineralisation at all time points compared to the rhGDF-5 group. These results also verify that there are distinct rates of bone tissue formation and maturation patterns by which bone defects heal depending upon the treatment.

It is important to note that the present study does not confirm an endochondral ossification path by means of the measured in vivo mechanical relaxation parameters for the rhBMP-2 treated group, as would have been suggested by some earlier studies (Yasko et al., 1992; Koempel et al., 1998; Einhorn et al., 2003; Edwards et al., 2004; Glatt et al., 2008; Yu et al., 2010). For instance, a study by Yasko et al. (1992) that used a rat model similar to ours, demonstrated by weekly histological examination that the healing of rat segmental defects occurs via endochondral ossification. Those studies used a demineralised rat-bone matrix (DBM) to deliver BMP-2, while our study used a Type 1 bovine collagen sponge. Although not able to induce healing by itself, DBM might have provided additional growth factors that contributed to a chondrogenic response during the healing. The fact is, in the present study, there wasn't even the slightest evidence to suggest that the cartilage was present as early as two week time point in the BMP-2 group. While in the study by Yasko et al. cartilaginous tissue persisted at least until 6 weeks post surgery. Of course, another contributing factor might have been the significantly higher dose of rhBMP2 used in our study. We used a 4.5 times higher dose of rhBMP-2 which might have changed not only the rate of bone repair, but also the biologic pathway through which bone tissues form in the early stage of defect healing. Moreover, since the dose of rhBMP-2 used was quite high, the equivalent dose for rhGDF-2 may not have been optimal, therefore, the differences observed during the defect healing could have been a dose effect related to the different receptor affinities for these growth factors. While these reports from different studies cannot be confirmed by the current or other studies, the question remains whether the pattern of bone formation and maturation in fact used different biological pathways with different proteins.

From the above discussion, it is evident that the newly developed in vivo biomechanical test, used in combination with in vivo radiological evaluation provides a robust approach to evaluate properties of bone tissue as defect healing progresses. The advantage of the new monitoring device over the other monitoring techniques is that it allows the sequential measurements of callus tissue and it facilitates direct comparison within the same animal at multiple time points. In addition, the in vivo monitoring of defect healing reduces the number of animals required and the variability between the treatment groups. More importantly, the in vivo biomechanical test permits temporal monitoring of callus tissue from the early stage of defect healing, at the time when radiological evaluation is not possible. Furthermore, longitudinal stiffness measurements and image monitoring, either at weekly or biweekly intervals, allow for a direct comparison of callus tissue maturation within the same sample population. Thus, the new monitoring device demonstrated its ability to monitor the process of bone formation and maturation in the in vivo defect model by clearly showing distinct healing rates in response to different BMPs. On the contrary, weekly measurements could have had an effect on the process of fracture healing. It is plausible that the mechanical stimulation, which occurred during the procedure used to measure callus stiffness, could have altered the patterns of defect healing. However, since all groups were exposed to the identical weekly biomechanical in vivo measurement regimen, the findings can be directly compared and confirmed that the different BMPs used had different rates of healing defects. Nevertheless, we are currently analysing the data to determine if the influence of mechanical stimulation had an effect on bone healing even though the mechanical parameters to measure tissue competence were reduced to a minimum.

In conclusion, this study confirms that the newly developed biomechanical test was able to monitor temporal changes of bone tissue formation and maturation as defect healing progressed in an in vivo rat model. Due to a lack of early time points in the histological analyses, the current study design cannot validate the distinct pathways of tissue regeneration and maturation during the initial phase of healing. Nevertheless, this monitoring method is the first that allows an in vivo detection of functional tissue maturation under different effects of treatments on bone healing in small animals. More importantly, the key finding of this experiment is that rhBMP-2 and rhGDF-5 treated defects, under the same conditions, have distinct rates of bone formation and maturation to heal bony defects. This knowledge should not only be useful when planning new experimental studies, but also when developing strategies for the regeneration of specific tissues treated under various clinical conditions in humans. Therefore, careful consideration of the specific biological treatment is required, and further studies are necessary to establish the mechanisms through which biology and other boundary conditions interact at the tissue and cellular level. We can anticipate that once these mechanisms are properly 
understood and tested in a larger species they could be translated into clinical practice for more efficient regeneration of bone tissue.

\section{Acknowledgments}

The authors would like to thank Carolin Schwarz for her assistance in conducting histological analysis and Bettina Willie for statistical assistance. This study was supported by a grant of the German Research Foundation (DFG SFB 760) and funding by the GSC 203 for Carolin Schwarz.

\section{References}

Bai X, Xiao Z, Pan Y, Hu J, Pohl J, Wen J, Li L (2004) Cartilage-derived morphogenetic protein-1 promotes the differentiation of mesenchymal stem cells into chondrocytes. Biochem Biophys Res Commun 325: 453460.

Beaupre GS, Hayes WC, Jofe MH, White AA, 3rd (1983) Monitoring fracture site properties with external fixation. J Biomech Eng 105: 120-126.

Claes L, Grass R, Schmickal T, Kisse B, Eggers C, Gerngross H, Mutschler W, Arand M, Wintermeyer T, Wentzensen A (2002) Monitoring and healing analysis of 100 tibial shaft fractures. Langenbecks Arch Surg 387: 146-152.

Edwards RB, 3rd, Seeherman HJ, Bogdanske JJ, Devitt J, Vanderby R, Jr, Markel MD (2004) Percutaneous injection of recombinant human bone morphogenetic protein-2 in a calcium phosphate paste accelerates healing of a canine tibial osteotomy. J Bone Joint Surg Am 86-A: 1425-1438.

Einhorn TA, Majeska RJ, Mohaideen A, Kagel EM, Bouxsein ML, Turek TJ, Wozney JM (2003) A single percutaneous injection of recombinant human bone morphogenetic protein-2 accelerates fracture repair. J Bone Joint Surg Am 85-A: 1425-1435.

Erlacher L, McCartney J, Piek E, ten Dijke P, Yanagishita M, Oppermann H, Luyten FP (1998a) Cartilage-derived morphogenetic proteins and osteogenic protein-1 differentially regulate osteogenesis. J Bone Miner Res 13: 383-392.

Erlacher L, Ng CK, Ullrich R, Krieger S, Luyten FP (1998b) Presence of cartilage-derived morphogenetic proteins in articular cartilage and enhancement of matrix replacement in vitro. Arthritis Rheum 41: 263-273.

Evans M, Kenwright J, Cunningham JL (1988) Design and performance of a fracture monitoring transducer. $\mathrm{J}$ Biomed Eng 10: 64-69.

Floerkemeier T, Hurschler C, Witte F, Wellmann M, Thorey F, Vogt U, Windhagen H (2005) Comparison of various types of stiffness as predictors of the load-bearing capacity of callus tissue. J Bone Joint Surg Br 87: 16941699.

Glatt V, Kwong FN, Park K, Parry N, Griffin D, Vrahas M, Evans CH, Harris MB (2009) Ability of rhBMP-2 to enhance bone healing in the presence of tobramycin:evaluation in a rat segmental defect model. J Orthop Trauma 23: 693-701.

Gruber R, Mayer C, Schulz W, Graninger W, Peterlik M, Watzek G, Luyten FP, Erlacher L (2000) Stimulatory effects of cartilage-derived morphogenetic proteins 1 and 2 on osteogenic differentiation of bone marrow stromal cells. Cytokine 12: 1630-1638.

Hente R, Cordey J, Perren SM (2003) In vivo measurement of bending stiffness in fracture healing. Biomed Eng Online 2: 8.

Hotten GC, Matsumoto T, Kimura M, Bechtold RF, Kron R, Ohara T, Tanaka H, Satoh Y, Okazaki M, Shirai T, Pan H, Kawai S, Pohl JS, Kudo A (1996) Recombinant human growth/differentiation factor 5 stimulates mesenchyme aggregation and chondrogenesis responsible for the skeletal development of limbs. Growth Factors 13: 65-74.

Kadomatsu H, Matsuyama T, Yoshimoto T, Negishi Y, Sekiya H, Yamamoto M, Izumi Y (2008) Injectable growth/ differentiation factor-5-recombinant human collagen composite induces endochondral ossification via Sryrelated HMG box 9 (Sox9) expression and angiogenesis in murine calvariae. J Periodontal Res 43: 483-489.

Kakudo N, Wang YB, Miyake S, Kushida S, Kusumoto K (2007) Analysis of osteochondro-induction using growth and differentiation factor-5 in rat muscle. Life Sci 81: 137143.

Koempel JA, Patt BS, O’Grady K, Wozney J, Toriumi DM (1998) The effect of recombinant human bone morphogenetic protein-2 on the integration of porous hydroxyapatite implants with bone. J Biomed Mater Res 41: 359-363.

Mehta M, Schell H, Schwarz C, Peters A, SchmidtBleek K, Ellinghaus A, Bail HJ, Duda GN, Lienau J (2011) A 5-mm femoral defect in female but not in male rats leads to a reproducible atrophic non-union. Arch Orthop Trauma Surg 131: 121-129.

Richardson JB, Cunningham JL, Goodship AE, O'Connor BT, Kenwright J (1994) Measuring stiffness can define healing of tibial fractures. J Bone Joint Surg Br 76: 389-394.

Roberts SG, Steele CR (2000) Efficacy of monitoring long-bone fracture healing by measurement of either bone stiffness or resonant frequency: numerical simulation. J Orthop Res 18: 691-697.

Rosen V (2006) BMP and BMP inhibitors in bone. Ann N Y Acad Sci 1068: 19-25.

Seide K, Weinrich N, Wenzl ME, Wolter D, Jurgens C (2004) Three-dimensional load measurements in an external fixator. J Biomech 37: 1361-1369.

Shimaoka H, Dohi Y, Ohgushi H, Ikeuchi M, Okamoto M, Kudo A, Kirita T, Yonemasu K (2004) Recombinant growth/differentiation factor-5 (GDF-5) stimulates osteogenic differentiation of marrow mesenchymal stem cells in porous hydroxyapatite ceramic. J Biomed Mater Res A 68: 168-176.

Spiro RC, Liu L, Heidaran MA, Thompson AY, Ng CK, Pohl J, Poser JW (2000) Inductive activity of recombinant human growth and differentiation factor-5. Biochem Soc Trans 28: 362-368. 
Thorey F, Richter A, Besdo S, Hackenbroich C, MeyerLindenberg A, Hurschler C, Windhagen H (2008) A new bending stiffness measurement device to monitor the influence of different intramedullar implants during healing period. Technol Health Care 16: 129-140.

Tsuji K, Bandyopadhyay A, Harfe BD, Cox K, Kakar S, Gerstenfeld L, Einhorn T, Tabin CJ, Rosen V (2006) BMP2 activity, although dispensable for bone formation, is required for the initiation of fracture healing. Nat Genet 38: 1424-1429.

Urist MR (1965) Bone: formation by autoinduction. Science 150: 893-899.

Wolfman NM, Hattersley G, Cox K, Celeste AJ, Nelson R, Yamaji N, Dube JL, DiBlasio-Smith E, Nove J, Song JJ, Wozney JM, Rosen V (1997) Ectopic induction of tendon and ligament in rats by growth and differentiation factors 5, 6, and 7, members of the TGF-beta gene family. J Clin Invest 100: 321-330.

Yasko AW, Lane JM, Fellinger EJ, Rosen V, Wozney JM, Wang EA (1992) The healing of segmental bone defects, induced by recombinant human bone morphogenetic protein (rhBMP-2). A radiographic, histological, and biomechanical study in rats. J Bone Joint Surg Am 74: 659-670.

Yu YY, Lieu S, Lu C, Colnot C (2010) Bone morphogenetic protein 2 stimulates endochondral ossification by regulating periosteal cell fate during bone repair. Bone 47: 65-73.

\section{Discussion with Reviewers}

Reviewer I: A much better control than a defect which has had $\mathrm{HCl}$ placed in it, would be sponge alone, as the natural healing pattern could be determined. Please comment.

Authors: It is possible that even a negligibly low concentration of $\mathrm{HCl}$ could have affected defect healing and hence could be considered as a treatment. However, this was not the case and the results from our study show that the "control" group had no defect healing or other abnormal tissue reaction in the defect gap at the end of the 6 week treatment as observed by histology. Furthermore, there is enough evidence in the literature (Einhorn et al., 1984; Yasko et al., 1992; Glatt et al., 2009, text references, and Betz et al., 2006; Betz et al., 2007a) and from experiments in our laboratory (unpublished data) confirming that critical size defects either left empty (natural pattern of healing) or loaded with a saline solution scaffold does not heal and require an intervention such as growth factors or other osteoinductive agents. Knowing this, we felt that there was no reason to have an empty control group, and more importantly, the intention of this study was not to determine the natural healing pattern of the defects. The main purpose of this study was to determine temporal changes and patterns of tissue formation and maturation by comparing two different growth factors: BMP-2 known to enhance osteogenesis and GDF-5 known to be involved in the chondrogenesis. The $\mathrm{HCl}$ loaded on the sponge instead of saline was chosen as a control for the GDF-5 group because $\mathrm{HCl}$ solution was needed to dissolve GDF-5 protein to make sure it is stable and folded right as recommended by the manufacturer $(10 \mathrm{mM} \mathrm{HCl}$, Biopharm). Therefore, to have a direct comparison of a scaffold loaded with and without GDF-5, we used the same solvent for both groups.

Reviewer I: You state that "in the rhBMP-2 group (Fig. $8 \mathrm{c}$ ), a thin new periosteal cortical callus bridged the defect spanning from distal to the proximal side, while a double cortical callus was seen on the opposite side" (Results, Histology). In how many cases? The same comment for the other treatments.

Authors: The described phenomenon with double cortical callus was seen for every single specimen in BMP-2 and GDF-5 groups (Fig. 9). Although in the GDF-5 group double cortices extended only on one side from the proximal edge of the defect towards femoral head. This double cortex formation is not surprising and has been documented in other studies. A study by Gerstenfeld et al. (Gerstenfeld et al., 2006) investigated 3D spatial fracture callus morphogenesis at different time points in a rat femur and tibia, and found that the outer cortical shell was formed as new bone grew over the cartilage and became the new periosteal margin adjacent to the muscle. The inner shell was formed by a new surface of bone that has developed on top of the original cortex and was part of the original periosteal response. In the longitudinal view, the edge of the callus was seen where the original bone bifurcates into two surfaces of bone. Another study by Isaksson et al. (2008) investigated remodelling of fracture callus in mice. Their experimental model also demonstrated formation of a double cortex during remodelling phase of healing. It developed at 28 days post fracture and was evident in all animals after 48 days of healing. The studies mentioned above are not the only ones that have reported double cortex callus formation (Desai et al., 2003; Meyer et al., 2003; Ashraf et al., 2007), therefore it appears that this is a normal event in rodents that undergo bone repair. To the authors' knowledge this phenomenon is specific to rodents, and does not occur in other species.

Reviewer I: Do you think a rodent model is appropriate for the assessment of growth factors, as the efficacy of growth factors like BMP-2 is known to reduce with higher order mammals?

Authors: Rodent models are extremely important for studying potential therapies for fracture healing, even though evidence in the literature clearly shows that the efficacy of treatments used from rodents to larger animal models and human clinical trials is reduced. Significant progress has taken place in our understanding of the molecular, cellular and tissue events in bone healing using rodent models. From this understanding, targets for therapeutic interventions such as BMPs have emerged in an attempt to treat malunions, nonunions and large bone defects. More recent developments in the mouse by Tsuji et al. (2006, text reference) used BMP-2 conditional knockout mouse model (in the limb) and found that fractures did not heal without the presence of endogenous BMP-2, despite that the other osteogenic stimuli such as BMP-4 and BMP-7 were present. This type of study could 

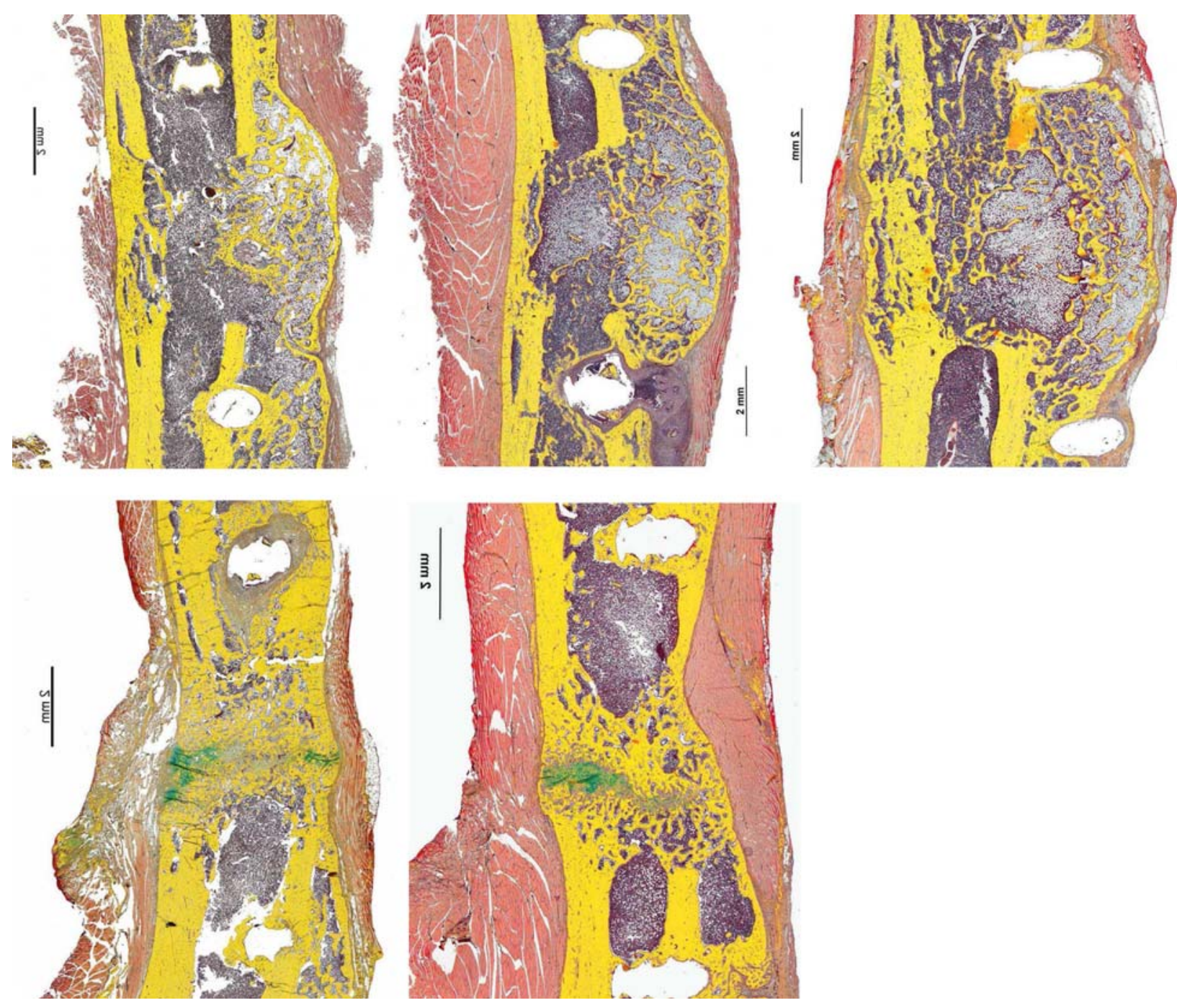

Fig. 9. Histological longitudinal sections stained with Movat Pentachrome from the femoral segmental defects 6 weeks post surgery treated with BMP-2 (top row) and GDF-5 (bottom row). Scale bars $=2 \mathrm{~mm}$.

have not been investigated in a rat of course, but even more so in the larger animal models. Understanding the mechanisms and genes responsible in fracture and bone defect healing has the potential to unravel why certain fractures or individuals fail to heal. Understanding these mechanisms can lead to the identification of new therapeutic strategies and molecular targets to improve and insure bone healing. Another important benefit using small rodent models such as rat and mice, is that it allows manipulation of molecular biology, use of greater number of animals in the experiments, are proven to be very reproducible and have potentially quicker healing rates. Moreover, there are greater availability of molecular screening tools, such as primers and antibodies, to analyze gene and protein expression levels in growth factor mediated pathways, which are not as widely available in the larger species. Furthermore, athymic mice and rats can accept human cells, factors and human tissues. In rodent models specific and conditional knockout studies also possible where targeted genes can be silenced to determine their importance and involvement in tissue regeneration. For the aforementioned reasons, rodent models are very important initial and the only possible model, to test new therapeutics before it can be confirmed in larger animals and eventually in human clinical trials. Of course which animal model is chosen is dependent upon the nature of the study and intervention analyzed. Nevertheless, for the initial preclinical studies to develop new pharmaceuticals like growth factors for potential treatment therapies in humans, rodents are a perfect choice before it can be validated in the larger species.

Reviewer II: In Fig. $8 \mathrm{~d}$ it is difficult to discern the bluegreen staining claimed by the authors.

Authors: In order to better demonstrate our observations of blue-green staining we add histological longitudinal sections (stained with Movat Pentachrome) from additional animals at three different magnifications ( $\mathrm{x} 5, \mathrm{x} 10$ and $\mathrm{x} 20$ ) adjacent to the defect edges of old cortical bone near the new bone interface and along the trabecular bone surface in the defect gap (Fig. 10). This blue-green staining most likely signifies remodelling phase in the bone tissue.

Reviewer II: Why are such high amounts $(50 \mu \mathrm{g})$ of BMP2 and GDF-5 used? This is much higher than most other investigators use, or can afford! 

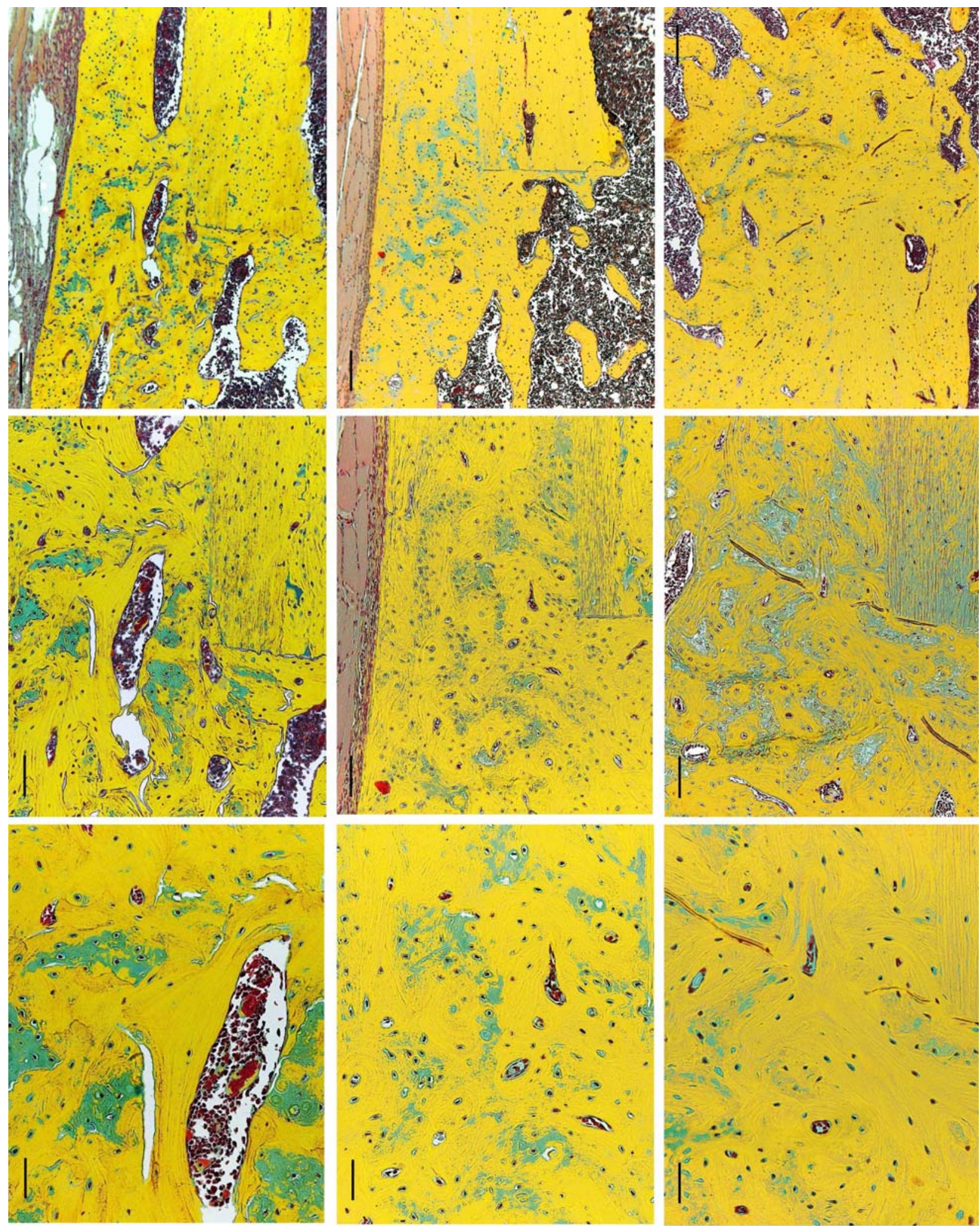

Fig. 10. Histological longitudinal sections (stained with Movat Pentachrome) from three additional animals at different magnifications (x5-top row, x10-middle row, x20-bottom row). Scale bar for top row $=1 \mathrm{~mm}$; middle row $=2 \mathrm{~mm}$; bottom row $=4 \mathrm{~mm}$.

Authors: The relatively high amount $(50 \mu \mathrm{g})$ of GDF-5 protein was used for this study, because we were unable to identify from the literature any experiments using an in vivo long bone femoral rat defect model where GDF-5 treatment was used to enhance bony healing. We selected a relatively high dose of protein for our initial in vivo experiment in order to ensure defect healing. Hence, a high dose of BMP-2 was also chosen so the collected data in this experiment could be directly compared among groups. Ongoing studies of the described defect model are trying to identify the most effective minimal dose required. 


\section{Additional References}

Ashraf N, Meyer MH, Frick S, Meyer RA Jr (2007) Evidence for overgrowth after midfemoral fracture via increased RNA for mitosis. Clin Orthop Relat Res 454: 214-222.

Betz OB, Betz VM, Nazarian A, Pilapil CG, Vrahas MS, Bouxsein ML, Gerstenfeld LC, Einhorn TA, Evans $\mathrm{CH}$ (2006) Direct percutaneous gene delivery to enhance healing of segmental bone defects. J Bone Joint Surg Am 88: $355-365$.

Betz OB, Betz VM, Nazarian A, Egermann M, Gerstenfeld LC, Einhorn TA, Vrahas MS, Bouxsein ML, Evans CH (2007) Delayed administration of adenoviral BMP-2 vector improves the formation of bone in osseous defects. Gene Ther 14: 1039-1044.

Desai BJ, Meyer MH, Porter S, Kellam JF, Meyer RA $\mathrm{Jr}$ (2003) The effect of age on gene expression in adult and juvenile rats following femoral fracture. J Orthop Trauma 17: 689-698.

Gerstenfeld LC, Alkhiary YM, Krall EA, Nicholls FH, Stapleton SN, Fitch JL, Bauer M, Kayal R, Graves DT, Jepsen KJ, Einhorn TA (2006) Three-dimensional reconstruction of fracture callus morphogenesis. J Histochem Cytochem 54: 1215-1228.

Isaksson H, Grongroft I, Wilson W, van Donkelaar CC, van Rietbergen B, Tami A, Huiskes R, Ito K (2008) Remodeling of fracture callus in mice is consistent with mechanical loading and bone remodeling theory. J Orthop Res 27: 664-672.

Meyer RA Jr, Meyer MH, Tenholder M, Wondracek S, Wasserman R, Garges P (2003) Gene expression in older rats with delayed union of femoral fractures. J Bone Joint Surg Am 85-A: 1243-1254. 